\title{
Crystal Structure of Human Enterovirus 71
}

\author{
Pavel Plevka ${ }^{1}$, Rushika Perera ${ }^{1}$, Jane Cardosa ${ }^{2}$, Richard J. Kuhn ${ }^{1}$, and Michael G. \\ Rossmann ${ }^{1, *}$ \\ ${ }^{1}$ Department of Biological Sciences, Purdue University, West Lafayette, Indiana 47907, USA \\ ${ }^{2}$ Sentinext Therapeutics, 10050 Penang, Malaysia
}

\begin{abstract}
Enterovirus 71 is a picornavirus associated with fatal neurological illness in infants and young children. Here we report the crystal structure of enterovirus 71 and show that, unlike in other enteroviruses, the "pocket factor", a small molecule that stabilizes the virus, is partly exposed on the floor of the canyon. Thus the structure of antiviral compounds may require a hydrophilic head group designed to interact with residues at the entrance of the pocket.
\end{abstract}

\begin{abstract}
Human enterovirus 71 (EV71) is a picornavirus that causes mild and self-limiting hand, foot, and mouth disease. Nevertheless, EV71 infections may progress to polio-like paralysis and fatal encephalitis in infants and young children (1). Large EV71 outbreaks have been reported in the Pacific region of Asia. The virus is considered a significant and emerging public health threat (2).

We have determined the crystal structure of EV71 to 3.8 $\mathrm{A}$ resolution [(3), table S1]. The EV71 virion, like other picornaviruses (4), has quasi-T $=3$ symmetry with 60 copies of each of the viral structural proteins VP1, VP2, VP3 that have $\beta$-sandwich "jelly-roll" folds. Additionally, 60 copies of a small protein VP4 are attached to the inner surface of the capsid (Fig. 1A). A common feature of enterovirus virions is a depression around the icosahedral five-fold axes called the "canyon" (4). Receptors that have an immunoglobulin-like fold bind in the canyon (5). This causes expulsion of a hydrophobic "pocket factor" that stabilizes the virion $(5,6)$ from a pocket in VP1 immediately below the floor of the canyon. The destabilization is a likely prelude to genome release. Antiviral compounds that bind into the pocket with high affinity are potent picornavirus inhibitors (5). The electron density of EV71 showed the presence of a pocket factor which was modeled as lauric acid (Fig. 1A). The height of the pocket factor electron density was comparable to that of the surrounding protein residues.
\end{abstract}

The most variable regions of picornavirus virions are the loops exposed on the virion surface that are also the most important neutralizing immunogenic sites $(4,7)$. The surface loops of VP1 are located around icosahedral fivefold axes and form the "northern" rim of the canyon (Fig. 1A). The canyon of EV71 is shallower than in most other enteroviruses because the EV71 VP1 loops are smaller (Fig. 1B). The open structure of the EV71 canyon indicates that it cannot provide immunological seclusion for the residues located at the bottom of the canyon and is thus less likely to serve as a binding site for receptors. Thus it is significant

\footnotetext{
*To whom correspondence should be addressed.mr@purdue.edu.

Supplementary Materials:

Materials and Methods

Table S1

References (10-16)
} 
that the molecules which have been identified as receptors of $\operatorname{EV71}(8,9)$ do not have immunoglobulin-like folds. In VP2 the most prominent surface loop is the "puff" that forms the "southern rim" of the canyon. The largest protrusion on the surface of the virus from VP3 is the "knob" (Fig. 1A). The puff and knob regions are involved in binding to nonimmunoglobulin-like receptors in some other picornaviruses. Lys 149 in the puff was shown to interact with P-selectin glycoprotein ligand-1, one of the two identified receptors of EV71 (9).

By analogy with other picornaviruses, the presence of the pocket factor in EV71 (Fig. 1C) indicates that it regulates the uncoating process. The EV71 pocket factor has about $40 \AA^{2}$ of surface accessible to solvent, whereas pocket factors in other enteroviruses are completely buried (Fig. 1D). Since the head of the EV71 pocket factor interacts with polar residues on the canyon floor, anti-EV71 compounds may require a hydrophilic head group. In contrast, inhibitors for other picornaviruses are completely hydrophobic and do not interact with residues forming the canyon floor. This information enables rational drug design of urgently needed EV71 therapeutics.

\section{Supplementary Material}

Refer to Web version on PubMed Central for supplementary material.

\section{Acknowledgments}

We thank APS (supported by the Department of Energy contract DE-AC02-06CH11357) staff, Vukica Srajer and Robert Henning of the BioCARS beamline 14 (NIH NCRR RR007707) and Robert Fischetti of GM/CA beamline 23. We thank Sheryl Kelly and Paul Chipman for help. Supported by NIH grant AI11219 to MGR. Coordinates were deposited with the Protein Data Bank as entry 4AED.

\section{References and Notes}

1. Alexander JP Jr, Baden L, Pallansch MA, Anderson LJ. Enterovirus 71 infections and neurologic disease--United States, 1977-1991. J. Infect. Dis. 1994; 169:905. [PubMed: 8133108]

2. Chan LG, et al. Deaths of children during an outbreak of hand, foot, and mouth disease in sarawak, malaysia: clinical and pathological characteristics of the disease. For the Outbreak Study Group. Clin. Infect. Dis. 2000; 31:678. [PubMed: 11017815]

3. For materials and methods, see supporting material available on Science Online

4. Rossmann MG, et al. Structure of a human common cold virus and functional relationship to other picornaviruses. Nature. 1985; 317:145. [PubMed: 2993920]

5. Rossmann MG, He Y, Kuhn RJ. Picornavirus-receptor interactions. Trends Microbiol. 2002; 10:324. [PubMed: 12110211]

6. Rossmann MG. Viral cell recognition and entry. Prot. Sci. 1994; 3:1712.

7. Sherry B, Mosser AG, Colonno RJ, Rueckert RR. Use of monoclonal antibodies to identify four neutralization immunogens on a common cold picornavirus, human rhinovirus 14. J. Virol. 1986; 57:246. [PubMed: 2416951]

8. Yamayoshi S, et al. Scavenger receptor B2 is a cellular receptor for enterovirus 71. Nat. Med. 2009; 15:798. [PubMed: 19543282]

9. Miyamura K, Nishimura Y, Abo M, Wakita T, Shimizu H. Adaptive mutations in the genomes of enterovirus 71 strains following infection of mouse cells expressing human P-selectin glycoprotein ligand-1. J. Gen. Virol. 2011; 92:287. [PubMed: 20943886] 
$A$

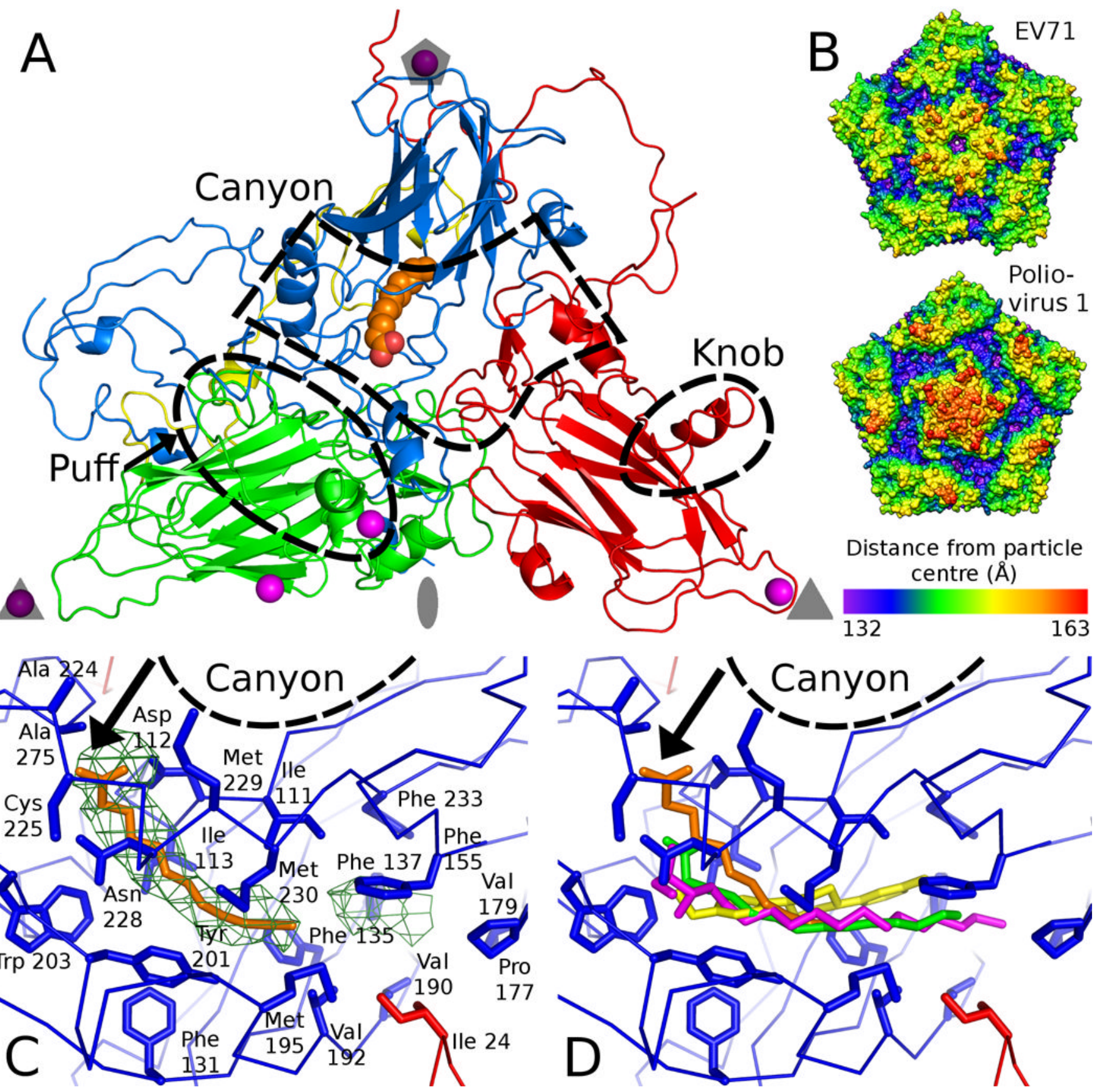

Fig. 1.

The EV71 capsid structure. (A) Cartoon diagram of EV71 icosahedral asymmetric unit showing VP1 (blue), VP2 (green), VP3 (red), VP4 (yellow) calcium ions (magenta) and the pocket factor (orange). The positions of the prominent capsid surface features are indicated by dashed lines. (B) Molecular surfaces of EV71 and Poliovirus of five icosahedral asymmetric units viewed down a fivefold axis. (C) The EV71 pocket factor electron density (green) was calculated by model-free phase extension (3). The main-chain is shown as lines connecting $\mathrm{Ca}$ atoms. Side-chains are displayed as stick models. (D) Positions of pocket factors in EV71 (orange), HRV1A (yellow), bovine enterovirus (green), and Poliovirus 1 (magenta). (C, D) The arrow shows the entrance to the pocket. 